Original Research Paper

\title{
Evaluation of Binary and Ternary Models in Powder Packing Density for Additive Manufacturing Applications
}

\author{
${ }^{1}$ Taher M. Abu-Lebdeh and ${ }^{2}$ Oluwatobiloba Adetokunbo Kalejaiye \\ ${ }^{1}$ Department of Civil Architectural and Environmental Engineering, North Carolina A\&T State University, Greensboro, NC \\ 27411, USA \\ ${ }^{2}$ Department of Mechanical Engineering, North Carolina A\&T State University, Greensboro, NC 27411, USA
}

\author{
Article history \\ Received: 19-04-2021 \\ Revised: 26-05-2021 \\ Accepted: 27-05-2021 \\ Corresponding Author: \\ Taher M. Abu-Lebdeh \\ Department of Civil, \\ Architectural and \\ Environmental Engineering, \\ North Carolina A\&T State \\ University, Greensboro, \\ NC27411, USA \\ Email: taher@ncat.edu
}

\begin{abstract}
Powder packing is a very important aspect of additive manufacturing (3-D printing), as it directly impacts the final physical and mechanical properties of the printed objects. Improving the packing density of powder directly impacts the microstructure of the finished 3D-printed product, which also contributes to the surface finish. In order to achieve the densest packing for a powder, different sizes of that powder must be mixed together in such a way that we minimize the voids, thereby increasing the density of the powder. To achieve this, a model that predicts the volume fraction of each powder grade becomes necessary to predict the maximum possible powder density. A wide variety of models have been developed for packing density which can be classified as: Binary Mixture Models, Ternary Mixture Models and Multi-Component Mixture Models. In this project, the Furnas ternary model and the modified Toufar binary model were used to evaluate the packing density of Boron powder. Two sets of Boron powders were used for the analysis: First set: $d_{10}=75 \mu \mathrm{m}, d_{50}=90 \mu \mathrm{m}, d_{90}=106 \mu \mathrm{m}$ and the second set: $d_{10}=3.11 \mu \mathrm{m}, d_{50}=14.0 \mu \mathrm{m}, d_{90}=30.2 \mu \mathrm{m}$. The three different sizes of each powder were randomly mixed to obtain the maximum packing density and compared with the theoretical packing density obtained by Furnas ternary model. The three sizes were then reduced to two sizes to obtain the packing density and compare the result with the modified Toufar binary model. Different mix rations were performed to evaluate the results obtained from both Furnas model and modified Toufar model. Results indicated that the models accurately predicted the maximum density of metal powder. Packing density obtained experimentally was $1.73 \mathrm{~g} / \mathrm{cm}^{3}$ at a mix ratio (coarse: Fine) of 89:11, while the values obtained by Furnas model and modified Toufar were 1.72 at $87: 13 \mathrm{mix}$ and $1.73 \mathrm{~g} / \mathrm{cm}^{3}$ at $89: 11$ mix respectively.
\end{abstract}

Keywords: Furnas Model, Ternary Mixture, Binary Mixture, Modified Toufar Model, Packing Density, Metal Powder, Additive Manufacturing

\section{Introduction}

The American Society for Testing and Materials (ASTM) defines Additive Manufacturing (AM) as "a process of joining materials to make objects from 3D model data, usually layer upon layer, as opposed to subtractive manufacturing technologies" (Frazier, 2014). Additive manufacturing allows for the making of very complex parts that might be difficult to fabricate using traditional methods. AM aims to reduce waste and has the potential of also reducing cost and carbon footprint.
According to the ASTM group "ASTM-F42-additive manufacturing," there are seven categories of Additive Manufacturing, namely: Binder Jetting; Material Jetting; Material Extrusion; Sheet Lamination; VAT Polymerization; Directed Energy Deposition; and Powder Bed Fusion. There are different kinds of Powder Bed Fusion techniques and these include: Direct Metal Laser Sintering (DMLS), Selective Laser Sintering (SLS), Selective Heat Sintering (SHS), Electron Beam Melting (EBM) and Selective Laser Melting (SLM). In the 3-D printing process, the design of the metal part to be 
additively manufactured is sent to the printer, these $3-\mathrm{D}$ printers consist of a powder bed and a roller, which spreads powder continuously. The printer now begins making the final product by printing the threedimensional object layer-by-layer from the bottom-up.

There are a wide variety of applications of additive manufacturing, which include (but limited to) ceramics, composites, metals, polymers and composite systems. Regardless of the application, the characteristics of the powder directly impact the outcome of the printing. Further, different types of powder materials adopt a default mechanism for packing and flow. There are a number of parameters that are used to classify powder and how it tends to arrange. There is a field of study dedicated to this, referred to as packing theory. There are number of factors that affect the packing density of a powder sample. These are: Particle morphology (shape and size of that particle); Powder flowability (angle of repose); particle size distribution (mixing different grades of powder can improve the packing density of the powder bed). It is known that the higher the packing density of the powder, the better the properties will be Akkurt et al. (2007; Koutný et al., 2016).

As aforementioned, improving the packing density of powder directly impacts the microstructure of the finished 3D-printed product, thus influencing the surface finish. To maximize the packing density of a particular type of powder, different sizes of that powder may be mixed to minimize the voids, regardless of the irregularities in the particle shape, viscosity, or flowability. One of the methods for increasing the packing density of powders is the formation of poly-fraction mixtures. In this method, the particle size of each subsequent fraction is smaller than the size of the voids formed by the preceding fraction. This means that the voids between larger particles will be filled by smaller particles thus decreasing the volume of voids and consequently increasing the packing density (Mangulkar and Jamkar, 2013; Khramtsov, 2009; Yu et al., 1992; Patankar and AN, 1980).

A model that predicts the volume or weight fraction of each powder grade is essential to predict the maximum possible powder density. The particle packing models evaluated in this project were: The Furnas model and the modified Toufar model. Furnas developed a method for predicting the volume fraction and weight fraction that gives the maximum density. Furnas utilized both binary and ternary mixtures (Furnas, 1931; Yu and Standish, 1991). Toufar et al. (1976) described a model to calculate the packing of binary mixtures for diameter ratios $0.22<d_{1} / d_{2}$ $<1.0$. Both the original Toufar model and the modified model can be used for estimating the packing of a multicomponent system (Johansen and Andersen, 1991).

\section{Particle Packing Models}

There are two categories of particle packing models: Discrete and continuous. Discrete models refer to packing of systems comprising two or more discrete size of powder particles. The coarsest powder particles form the base structure in which smaller powder particles fill the voids in the structure. Discrete models are based on the theory that each class of particle will pack to its maximum density in the available volume. There are three types of discrete models: Binary, ternary, multimodal mixture model (Mangulkar and Jamkar, 2013; Furnas, 1931; Wong and Kwan, 2014.

A binary model was postulated by Furnas. He performed experiments using spherical particles on the assumption that small particles fill the cavities between the large particles without disturbing the packing of the large particles. The mixture that Furnas studied consisted of coarse and fine material. He considered two cases defined by the volume fraction, $y$ of fine and coarse aggregate: Fine grain dominant (when the volume fraction of small particles is large, $y_{2} \gg y_{1}$ ) and coarse grain dominant (when the volume fraction of coarse particles is large, $y_{2} \gg y_{1}$ ). The two cases are viable if the particle diameters $d_{1} \ll d_{2}$. The packing density of the binary mixture also depends on the diameter ratio, $\frac{d_{1}}{d_{2}}$. If $\frac{d_{1}}{d_{2}} \approx$ 1 or $d_{1} \approx d_{2}$, an interaction effect arises which could form wall, loosening or wedging effects as seen in Fig. 1.

The wall effect happens when there is a looser packing density near the walls/edge of a container. The loosening effect is a result of fine particles being trapped among coarse particles while the wedging effect occurs when coarse particles are trapped among fine particles and leaves voids (Mangulkar and Jamkar, 2013; Furnas, 1931; Wong and Kwan, 2014).

Toufar et al. (1976) developed a model that calculates the packing density of binary mixtures for diameter ratios $0.22<\frac{d_{1}}{d_{2}}<1.0$. The theory of the model is that for diameter ratios $>0.22$ the smaller particles with diameter $d_{1}$ will be too large to be filled within the interstices between the larger particles with diameter $d_{2}$. The Toufar model also considered the statistical probability of the number of interstices between the coarser particles that are free from smaller particles (Fennis-Huijben, 2010). Toufar's model was modified for a shape factor by Goltermann et al. (1997). The modified Toufar model can predict the packing density of a mixture consisting of two monosized particle classes.

An extension of the binary models is the ternary models. Ternary models have been postulated which are based upon the fundamental principle of mixing three powders (Coarse, medium, fine) in a particular ratio. It was utilized by using the binary mixture model to calculate the packing density of ternary mixtures in relation to the weighted average of the total number of 
binary mixtures for diameters of specific ratios. Series of equations were often utilized to calculate possible weight mix ratio or volume ratio that will give the maximum packing density. The coarse powder particles usually form the base structure, the middle powder particle fill the available voids in the structure, while the fine particle fill the remaining void left by the middle particle. The multimodal model is based on the principle of mixing powder particles of different sizes. The coarsest powder usually forms the base structure while the other powders fill in the void left by latter in that other until there are no more voids. Theoretically, packing efficiency close to one hundred percent can be achieved but empirically, it is impossible due to the minute volume of powders that is required to fill successive voids left (Kumar et al., 2003).

Continuous model is a form of discrete approach that has adjacent sizes of class ratio that approach the ratio 1:1. Gaps are absent between size classes. Continuous approach assumes that all possible sizes exist in the particle distribution system. This system utilizes series of complex formulas. The wall effect, loosening effect and compaction effect do not affect its packing (Kumar et al., 2003; Furnas, 1931; Wong and Kwan, 2014).

\section{Factors Affecting the PACKING Density of Powder}

Particle properties that affect the packing density of powders include particle morphology (particle size, particle shape and convexity), particle density, particle size distribution, aspect ratio, rolling resistance and compressibility. The desirable particle shape is spherical. This is because it allows for high flowability during additive manufacturing. The particle size distribution has a significant effect on the packing density with narrow distribution resulting in lower packing densities than wide distributions. A wide particle size distribution allows the smaller powder to settle in between larger particles and reduce the voids. Figure 2 shows the different possible powder particle shapes of metal powders (Chateau, 2012).
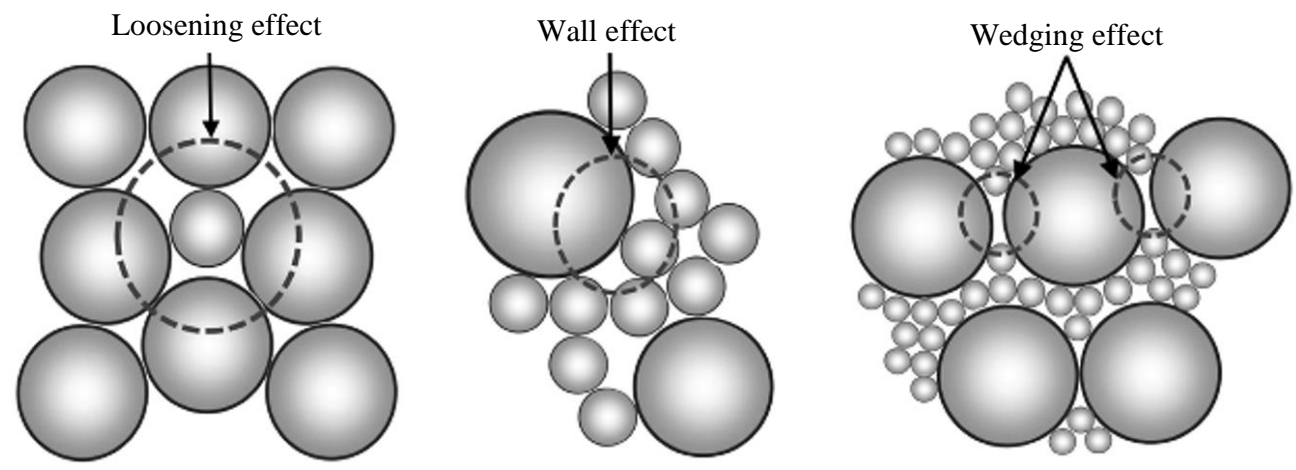

Fig. 1: Schematic representation of particle interactions (Wong and Kwan, 2013)

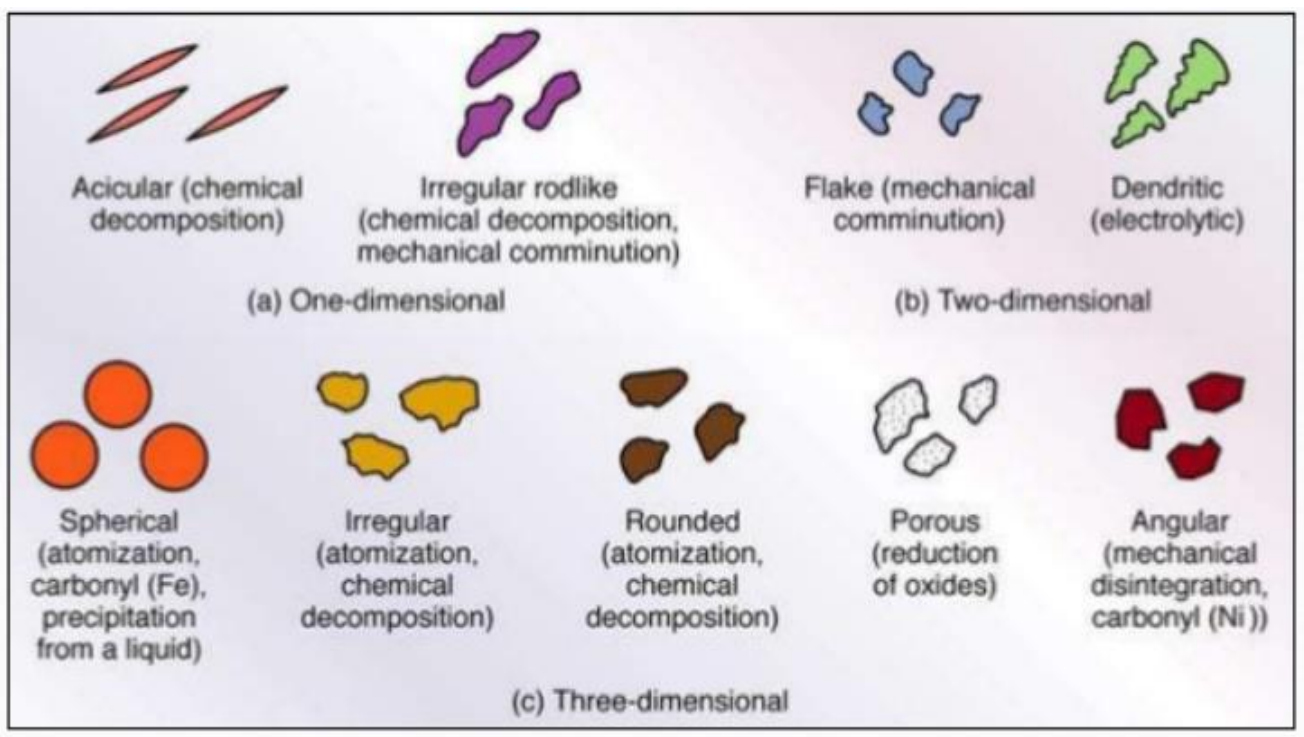

Fig. 2: Particle shapes in metal powder Rejeesh C. R 


\section{Methodology}

\section{Ternary Model}

Consider the first set, Boron powder was sieved into two sizes using sonic sifter separator. The sizes are 106 $\mu \mathrm{m}$ and $75 \mu \mathrm{m}$. With the diameters of the coarse powder, $d_{1}(106 \mu \mathrm{m})$ and fine powder $d_{3}(75 \mu \mathrm{m})$ known, an approximate match for the middle powder, $d_{2}(90 \mu \mathrm{m})$ was chosen according to Eq. 1:

$$
d_{2}=\sqrt{d_{1} * d_{3}}
$$

For the second set of Boron powder, the results obtained from the particle size analyzer are as follows: $d_{10}$ $(3.11 \mu \mathrm{m}), d_{50}(14.0 \mu \mathrm{m})$ and $d_{90}(30.2 \mu \mathrm{m})$. Three sizes of particles are needed to execute the ternary model. d50 was chosen from the first set and second set of the Boron powder. Since the smallest sieve size available in our lab is $25 \mu \mathrm{m}$, thus the $\mathrm{d} 50$ for the second set of Boron powder was chosen as $25 \mu \mathrm{m}$. Figure 3 shows the flow chart of the processes involved in the determination of both density and packing density of a ternary mixture. The methodology comprises eight steps as shown in Fig. 3: Particle size determination, bulk and tap volumes, void volume, weight ratio, determination of volume ratio, determination of density and determination of packing density.

\section{Particle Size Determination}

A Sonic Sifter Separator (L3P Sonic Sifter Separator, Avantech) is a precision instrument for the swift separation of a wide selection of dry particles and powders into fine micron range. It was used to sieve boron powder into different diameters. From equation 1, three different sizes were stacked into the machine. About $10 \mathrm{~g}$ of boron powder was put into the top cone of a sonic sifter. When the machine was turned on, a vertical column of air was created to oscillate through the set of sieves. The air motion alternately lifts the boron sample and then allows it to pass through the apertures of the sieve. The oscillation amplitude was varied to maximize the amount of boron powder passing through the sieves. A vertical mechanical pulse was applied to the sieves at regular intervals to break down any clustered particles and to help reduce any clogging of the sieves. Figure 4 shows the Avantech L3P Sonic Sifter Separator Mastersizer 3000 Particle size analyzer.

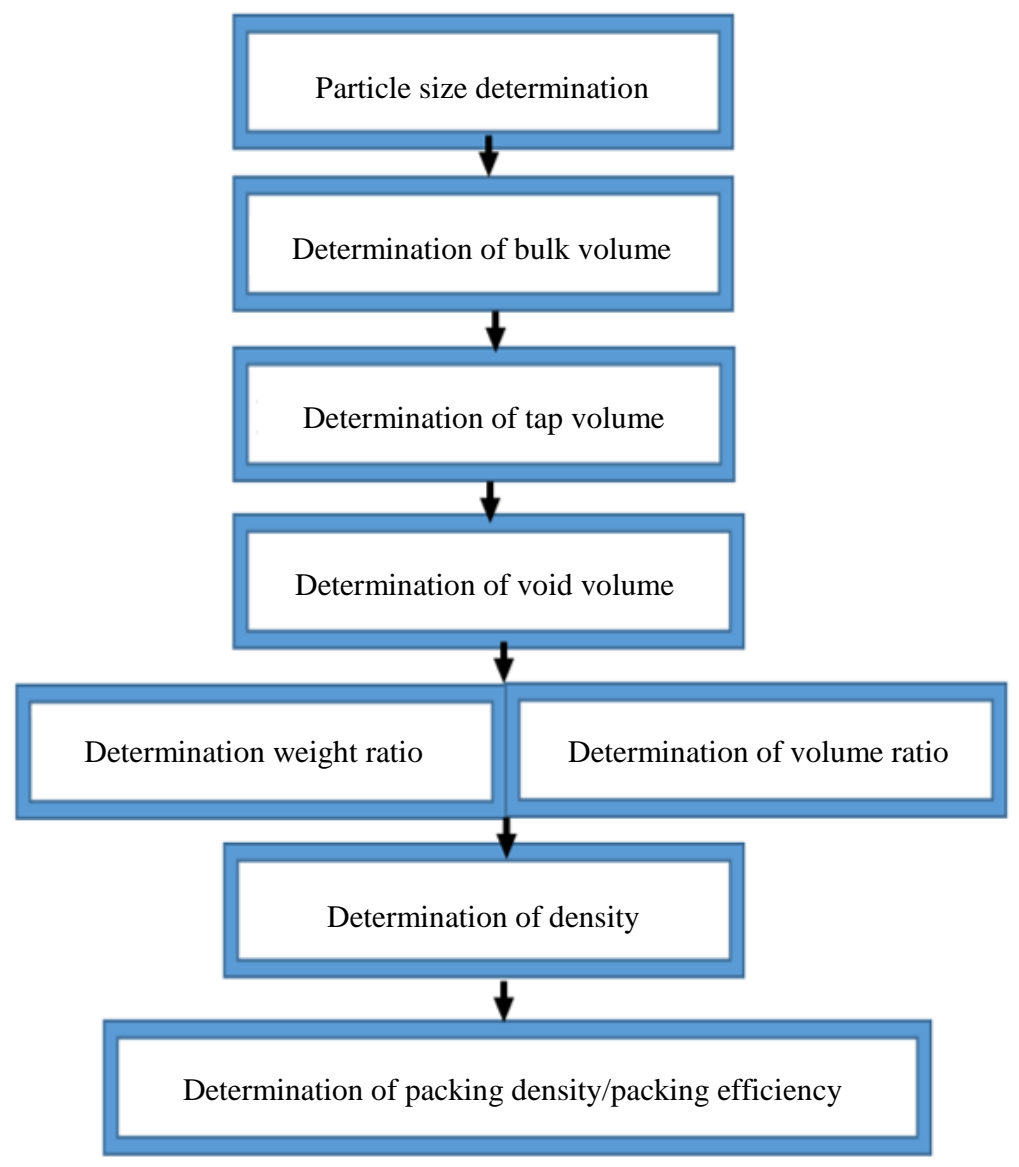

Fig. 3: Flow chat of the processes involved in the determination of both density and packing density of ternary mixture 

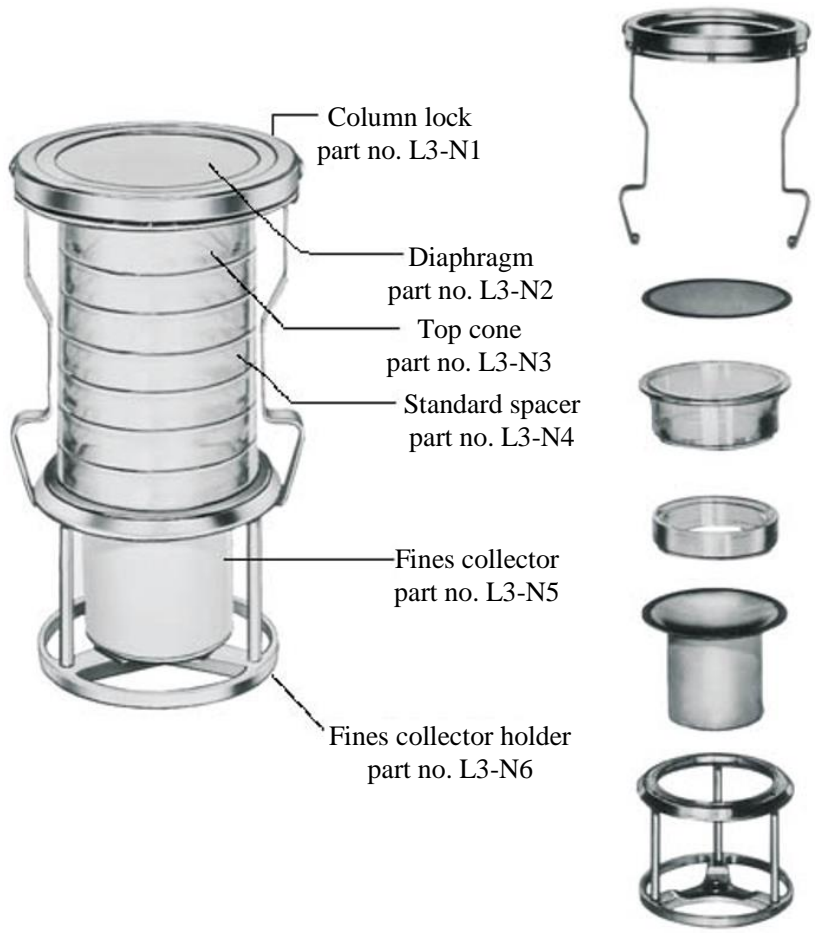

Fig. 4: L3P Sonic Sifter Separator (Avantech)

\section{Determination of Bulk Volume Tap Volume and Void Ratio}

A graduated cylinder was used to measure both the bulk volume and tap volume of each of the three sizes of the powders selected. Approximately $10 \mathrm{~g}$ of the selected powder size were measured with a balance (Mettler AE m b 163) and poured gently into the graduated cylinder. This is to obtain an accurate bulk volume. The bulk volume was then measured at eye level to reduce parallax error. The procedure was repeated 5 times for each powder and both the average and standard deviation were calculated. Each of the powders in the graduate cylinder was then tapped 250 times. The tap volume was then recorded. The procedure was also repeated 5 times and both the average and standard deviation were calculated.

\section{Resutls and Discussion}

In this section, the results of the Furnas model and modified Toufar model are explained.

\section{Furnas Model}

The diameter of the coarse component is $d_{1}=90 \mu \mathrm{m}$, while the diameter of fine component is $d_{3}=25 \mu \mathrm{m}$. When these values were substituted into equation $1, d_{2}$ of the medium size was obtained to be $47 \mu \mathrm{m}$. The sieve diameter with the nearest diameter to $47 \mu \mathrm{m}$ is $45 \mu \mathrm{m}$. Bulk volume, tap volume, and void ratio were determined as described above and the results are tabulated in Table 1.

\section{Weight and Weight Fraction}

The total weight percentage is obtained from Eq. 2.

$W=W_{1}+1-W_{1}+\left(1-W_{1}\right) \frac{1-W_{2}}{W_{2}}$

It is made up of three parts: $W_{1}, 1-W_{1}$ and $\left(1-W_{1}\right)$ $\frac{1-W_{2}}{{ }_{W}}$. The first part, $W_{1}$, is the weight percentage of the coarse powder $\left(d_{1}\right)$, the second part, $1-W_{1}$, is the weight percentage of the middle powder $\left(\mathrm{d}_{2}\right)$ while the third part, $\left(1-W_{1}\right) \frac{1-W_{2}}{{ }_{W} 2}$, is the weight percentage of the fine powder $\left(\mathrm{d}_{3}\right) . W_{1}$ and $W_{2}$ are calculated from the following equations:

$W_{1}=\frac{\left(1-e_{1}\right) S_{1}}{\left(1-e_{1}\right) S_{1}+e_{1}\left(1-e_{2}\right) S_{2}}$

$W_{2}=\frac{\left(1-e_{2}\right) S_{2}}{\left(1-e_{2}\right) S_{2}+e_{2}\left(1-e_{3}\right) S_{3}}$

where, $S_{1}, S_{2}, S_{3}$ are the specific gravity of the coarse, middle and find powder, respectively. $e_{1}, e_{2}, e_{3}$ are the void ratio of the coarse, middle and fine powder, respectively. Void ratio $(e)$ is the ratio of the volume of void $\left(V_{v}\right)$ to the total or bulk volume $\left(V_{b}\right)$ : 
$e=\frac{V_{v}}{V_{b}}$

Substitute the values of specific gravities and void ratios into equations 3 and 4 yield $W_{1}=0.8442 \pm 0.15$ and $W_{2}=0.880509 \pm 0.12$. From equation 2 , Total weight percentage $=(0.8442)+(0.1558)+(0.02114)=1.02114$ with weight percentage of 82.67: 15.26: $2.07 \%$.

Now, if each smaller-sized particle exactly fills the voids created by the (larger) previously packed larger particle component, the total the final volume of the system will be the volume of the first component (largest sized particles). The reduction in volume as a result of filling the voids of component 1 with components 2 and 3 is equivalent to the total volume minus the volume of the first component. This will be the ideal case. However, for an actual system, the decrease in volume will be less than the ideal case, as components 2 and 3 may not exactly fill the voids created by component 1 . This decrease in volume $(\mathrm{y})$ is expressed as:

$y=1.0-2.62 K^{1 / 2}+1.62 K^{2 / n}$

$K=\frac{d_{n+1}}{d_{1}}$

The ratio between the smallest and large sizes, $K$ is obtained from Eq. 7 while the ratio of decrease in total volume, y is obtained from Eq. 7. The interval $n+1$ is the total number of sizes. From Eq. 7, $\mathrm{K}$ is 0.278 and from Eq. $6, y$ is 0.3086 .

The theoretical density of ternary mixture, $\rho$ is shown in Eq. 8 and the empirical density is shown in Eq. 9:

$\rho=\frac{\left(1-e_{1}\right) S_{1}+e_{1} S_{2}\left(1-e_{2}\right)+e_{1} e_{2} S_{3}\left(1-e_{3}\right)}{1+e_{1}\left(1+e_{2}\right)(1+y)}$

$\rho=\frac{\text { Total mass of mixture }}{\text { Total solid volume of mixture }}$

From Eq. 8, theoretical density, $\rho$ is $1.79 \pm 0.1012$ $\mathrm{g} / \mathrm{cm}^{3}$ while the empirical density shown in equation 9 is $1.76 \mathrm{~g} / \mathrm{cm}^{3}$ (1.7\% difference)

The packing density $(\phi)$ of a powder sample is the ratio of the solid or true volume $\left(V_{s}\right)$ to the total or bulk volume $\left(V_{b}\right)$ of the sample. Packing efficiency is derived by multiplying packing density by 100 . The empirical packing density is given by Eq. 10 which can be simplified to obtain Eq. 11. Theoretical packing density is given by Eq. 12:

$\phi=\frac{\text { Solid volume }}{\text { Bulk volume }}$
$\phi=\frac{V_{s}}{V_{b}}=\frac{V_{s}}{V_{s}+V_{v}}=\frac{V_{b}-V_{v}}{V_{b}}=\frac{V_{b}}{V_{b}}=\frac{V_{v}}{V_{b}}=1-e$

$\phi=\frac{1-e_{1}+e_{1}(1-y)\left(1-e_{2} e_{3}\right)}{1+e_{1}\left(1+e_{2}\right)(1-y)}$

From Eq. 12, theoretical packing density is $0.81 \pm 0.011$. Thus, the theoretical packing efficiency is $81 \%$. From equation 10 , the empirical packing density $(\phi)$ is 0.78 , thus the empirical packing efficiency is $78 \%$.

\section{The Modified Toufar Model}

Toufar's model (Eq. 13-17) calculates the packing density of binary mixtures. The concept of the model is that the smaller particles are packed in the voids of larger particles and so on. However, the model is limited to diameter ratios $0.22<\mathrm{d} 1 / \mathrm{d} 2<1.0$. However, for diameter ratios $>0.22$ the smaller particles will be too large to be placed within the voids between the larger particles. Therefore, the packing density should depend on the diameter ratio of the two particle classes, as expressed by the factor $k_{d}$ in equation 14 . Furthermore, the model assumes that each of the fine particles is placed between exactly four of the coarse particles, led to the shape factor $k_{s}$ which was later modified by Goltermann et al. (1997) as described in equations 15-17. The input parameters are the packing density and the amount and particle size of the particle groups used in a powder mixture. The output is the theoretical packing density of the mixture. For mixture optimization, the packing density of several mixture compositions need to be determined until the maximum packing density is found.

$$
\begin{aligned}
& \alpha_{t}=\frac{1}{\frac{r_{1}}{\alpha_{1}}+\frac{r_{2}}{\alpha_{2}}-r_{2}\left(\frac{1}{\alpha_{2}}-1\right) k_{d} k_{s}} \\
& k_{d}=\left(\frac{d_{2}-d_{1}}{d_{1}+d_{2}}\right) \\
& X=\frac{r_{1}}{r_{2}} \frac{\alpha_{2}}{\alpha_{1}\left(1-\alpha_{2}\right)} \\
& k_{s}=\frac{0.3881 x}{0.4753} \text { for } \times<0.4753 \\
& k_{s}=1-\frac{1+4 x}{(1+x)^{4}} \text { for } \times \geq 0.4753
\end{aligned}
$$

Where:

$k_{d}=$ The diameter ratio

$k_{s}=$ A shape effect

$r_{j}=$ The volume fraction of size class $j$

$\alpha_{t}=$ The packing density of the mixture 
Two sizes of Boron powder were used to evaluate and implement Toufar model. First set: $d_{10}=75 \mu \mathrm{m}, d_{50}=90$ $\mu \mathrm{m}, d_{90}=106 \mu \mathrm{m}$. The second set consists of: $d_{10}=3.11$ $\mu \mathrm{m}, d_{50}=14.0 \mu \mathrm{m}, d_{90}=30.2 \mu \mathrm{m}$. $\mathrm{d}_{50}$ was chosen from both Boron powders. The bulk and tap volumes of each powder were determined as described earlier. The bulk and solid volume of the fine powder $(14 \mu \mathrm{m})$ were 14.16 $\mathrm{cm}^{3}$ and $8.36 \mathrm{~cm}^{3}$ respectively. The packing density of the fine powder is then $8.36 / 14.16=0.5904$. Similarly, the bulk and solid volume of the coarse powder $(90 \mu \mathrm{m})$ were $9.58 \mathrm{~cm}^{3}$ and $7.8 \mathrm{~cm}^{3}$ respectively. Thus, the packing density of the coarse powder is $7.8 / 9.58=0.8142$. Table 2 shows the results obtained by applying Eq. 13-17. According to Toufar, the mix ratio with the highest packing density is the one that produces the highest density. From Table 2, the highest packing density $\left(\alpha_{t}\right)$ is 0.8458 at a mix ratio (coarse: Fine) of $89: 11$. The density of this binary mixture was measured and found to be equal to $1.73 \mathrm{~g} / \mathrm{cm} 3$.

Returning to Furnas ternary model, the maximum density of $1.79 \pm 0.1012 \mathrm{~g} / \mathrm{cm} 3$ was achieved at a volume fraction of coarse $(90 \mu \mathrm{m})$ : Medium $(45 \mu \mathrm{m})$ : Fine $(25$ $\mu \mathrm{m})$ of 82.67: 15.26: $2.07 \%$. \%. However, in order to compare with the Toufar binary model, the percentage will need to be converted to two mix ratios. To do that, $20 \mathrm{~g}$ of coarse powder was sieved in the medium size of $45 \mu \mathrm{m}$ sieve. $6.75 \mathrm{~g}$ powder particle passed through the sieve. Again, $20 \mathrm{~g}$ of fine powder was sieved in a $45 \mu \mathrm{m}$ sieve. All the powders went through. This means that the medium size $(45 \mu \mathrm{m})$ which represents $15.26 \%$ of ternary mixture contains $3.85 \%$ coarse $(6.75 / 26.75) * 15.26 \%$ and $11.41 \%$ fine $(20 / 26.75) * 15.26 \%$. If the split values are added to the coarse and fine, we have: Coarse: Fine $=$ $(82.67+3.85):(2.07+11.4)=87: 13$. This compares well with Toufar results of $89: 11$.

To validate both models, several random mixtures were prepared at different mix ratios and tested for their densities. As shown in Table 3, the maximum densities were achieved at mixture ratios found by both models. This confirms the validity of the models.

Table 1: Experimental Results of the first set

\begin{tabular}{llll}
\hline Name of Powder & Boron powder (Coarse) & Boron powder (Middle) & Boron powder (Fine) \\
\hline Diameter $\left(d_{n}\right), \mu \mathrm{m}$ & 90 & 45 & 25 \\
Bulk Volume $\left(V_{b}\right), \mathrm{cm}^{3}$ & $9.58 \pm 0.17$ & $9.28 \pm 0.22$ & $14.16 \pm 0.11$ \\
Tap Volume $\left(V_{s}\right), \mathrm{cm}^{3}$ & $7.80 \pm 0.14$ & $7.54 \pm 0.11$ & $8.36 \pm 0.11$ \\
Volume of void $\left(V_{v}\right), \mathrm{cm}^{3}$ & $1.78 \pm 0.18$ & $1.74 \pm 0.25$ & $5.80 \pm 0.16$ \\
Specific Gravity, $\mathrm{S}$ & 2.34 & 2.34 & 2.34 \\
Void ratio/void percentage $(e)$ & $0.185 \pm 0.10$ & $0.187 \pm 0.15$ & $0.41 \pm 0.03$ \\
\hline
\end{tabular}

Table 2: Results of the Modified Toufar Model

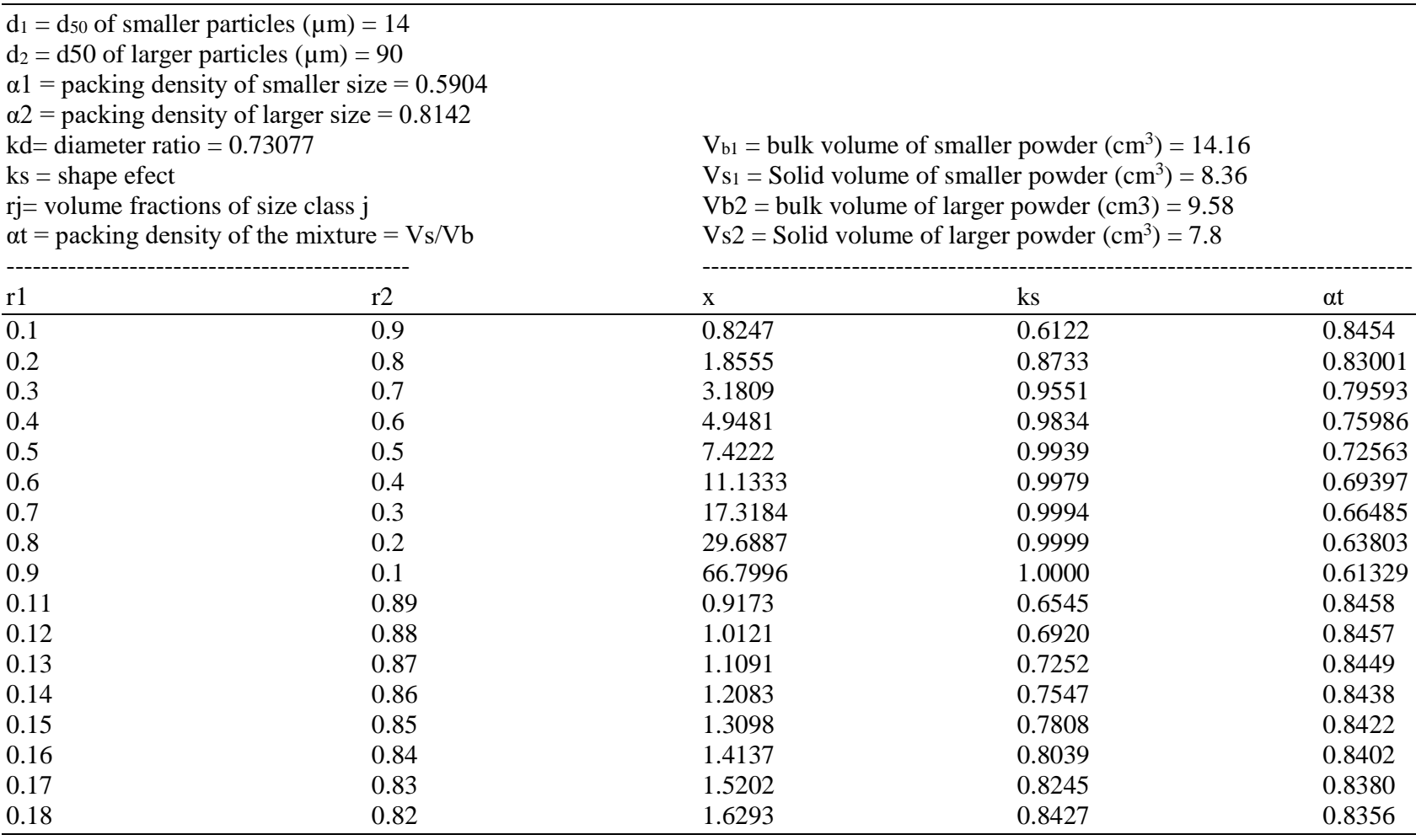


Table 3: Density Results from Furnas, Toufar and Random mix

\begin{tabular}{llll}
\hline & Method & $\%$ Mix ratio & Density $\left(\mathrm{g} / \mathrm{cm}^{\wedge} 3\right)$ \\
\hline 1 & Furnas (Experimental) & $83: 15: 2$ & 1.76 \\
2 & Furnas (Theoretical ternary mixture) & $83: 15: 2$ & 1.79 \\
3 & Furnas (Binary, experimental) & $87: 13$ & 1.72 \\
4 & Toufar (Binary, experimental) & $89: 11$ & 1.73 \\
5 & Random mix ratio & $95: 5$ & 1.69 \\
6 & Random mix ratio & $90: 10$ & 1.73 \\
7 & Random mix ratio & $80: 20$ & 1.61 \\
8 & Random mix ratio & $60: 40$ & 1.54 \\
9 & Random mix ratio & $50: 50$ & 1.48 \\
10 & Random mix ratio & $40: 60$ & 1.40 \\
11 & Random mix ratio & $30: 70$ & 1.32 \\
12 & Random mix ratio & $20: 80$ & 1.18 \\
13 & Random mix ratio & $10: 90$ & 1.02 \\
\hline
\end{tabular}

\section{Conclusion}

This work focused on the evaluation and implementation of Furnas ternary model and modified Toufar binary model to determine the maximum packing density as well as the volume fractions of a mixture's sizes to achiev the required packing. The following can be drawn from the results of this study:

1. The results obtained by Furnas ternary model and the modified Toufar binary model are not too far apart. It can be concluded that Furnas equations that determine the maximum density and volume fractions of ternary mixture appears to agree with experiments. Similarly, results obtained by modified Toufar model agree well with the experimental results for binary mixtures. Packing density obtained experimentally was $1.73 \mathrm{~g} / \mathrm{cm}^{3}$ at a mix ratio(coarse: Fine) of $89: 11$, while the values obtained by Furnas model and modified Toufar were 1.72 at 87:13 mix and $1.73 \mathrm{~g} / \mathrm{cm}^{3}$ at $89: 11$ mix respectively

2. Both, Furnas model and Toufar model (especially the modified Toufar model) can be used for binary and also for ternary systems

3. Both models are based on the concept that smaller particles are packed in the voids of larger particles and so on. This way, the particle packing models are suitable to calculate the packing density of powders based on a particle size distribution

4. The modified Toufar model is well suited to predict the packing density of a mixture consisting of two monosized particle classes. The calculation includes the size and amounts of particles in the mixture. Shape effects are indirectly taken into account via the packing density of the size classes

5. The Furnas model can predict the packing density of a ternary mixture consisting of three monosized particle classes. The calculation includes the density and void ratio of particles in the mixture. Size and shape effects are considered in the model
6. Future work should include ceramics and other powders for additive manufacturing.

\section{Author's Contributions}

Oluwatobiloba Adetokunbo Kalejaiye: Performed the experiments, analysed the data and participated in writing the manuscript.

Taher Abu-Lebdeh: Provided the research topic, guided the research development and supervised the entire work, also participated in writing the manuscript.

\section{Ethics}

This article is an original research paper. There are no ethical issues that may arise after the publication of this manuscript.

\section{References}

Akkurt, S., Romagnoli, M., \& Sütçü, M. (2007). DOE and ANN models for powder mixture packing. https://openaccess.iyte.edu.tr/xmlui/handle/11147/2094

Chateau, X. (2012). Understanding the Rheology of Concrete.1st Edition, Woodhead Publishing.

Fennis-Huijben, S. A. A. M. (2010). Design of ecological concrete by particle packing optimization. SAAM Fennis-Huijben.

Frazier, W. E. (2014). Metal additive manufacturing: a review. Journal of Materials Engineering and Performance, 23(6), 1917-1928. https://doi.org/10.1007/s11665-014-0958-z

Furnas, C. C. (1931). Grading aggregates-I.-Mathematical relations for beds of broken solids of maximum density. Industrial \& Engineering Chemistry, 23(9), 1052-1058. https://doi.org/10.1021/ie50261a017

Goltermann, P., Johansen, V., \& Palbøl, L. (1997). Packing of aggregates: an alternative tool to determine the optimal aggregate mix. Materials Journal, 94(5), 435-443. https://doi.org/10.14359/328 
Johansen, V., \& Andersen, P. J. (1991) Particle Packing and Concrete Properties. In: Skalny, J. and Mindess, S. (eds). Materials science of concrete 2. Westerville: American Ceramic Society.

Khramtsov, V. D. (2009). The packing density of the particles in powder mixtures of different dispersities. Russian Journal of Non-Ferrous Metals, 50(3), 294-297. https://doi.org/10.3103/S1067821209030213

Koutný, O., Kratochvíl, J., Švec, J., \& Bednárek, J. (2016). Modelling of packing density for particle composites design. Procedia Engineering, 151, 198-205. https://doi.org/10.1016/j.proeng.2016.07.386

Kumar, S. E. N. T. H. I. L., \& Santhanam, M. (2003). Particle packing theories and their application in concrete mixture proportioning: A review. Indian Concrete Journal, 77(9), 1324-1331.

Mangulkar, M. N., \& Jamkar, S. S. (2013). Review of particle packing theories used for concrete mix proportioning. Contributory Papers, 141.
Patankar, A. N., \& AN, P. (1980). XI: The packing of some non-spherical solid particles. https://pascalfrancis.inist.fr/vibad/index.php?action=getRecordDe tail\&idt=PASCAL8060414953

Toufar, W. (1976). Beitrag zur optimierung der packungsdichte polydisperser koerniger systeme. https://pascal-

francis.inist.fr/vibad/index.php?action=getRecordDe tail\&idt=PASCAL7770103111

Wong, V., \& Kwan, A. K. H. (2014). A 3-parameter model for packing density prediction of ternary mixes of spherical particles. Powder technology, 268, 357-367. https://doi.org/10.1016/j.powtec.2014.08.036

$\mathrm{Yu}$, A. B., \& Standish, N. (1991). Estimation of the porosity of particle mixtures by a linear-mixture packing model. Industrial \& Engineering Chemistry Research, 30(6), 1372-1385. https://doi.org/10.1021/ie00054a045

Yu, A. B., Zou, R. P., \& Standish, N. (1992). Packing of ternary mixtures of nonspherical particles. Journal of the American Ceramic Society, 75(10), 2765-2772. https://doi.org/10.1111/j.1151-2916.1992.tb05502.x 